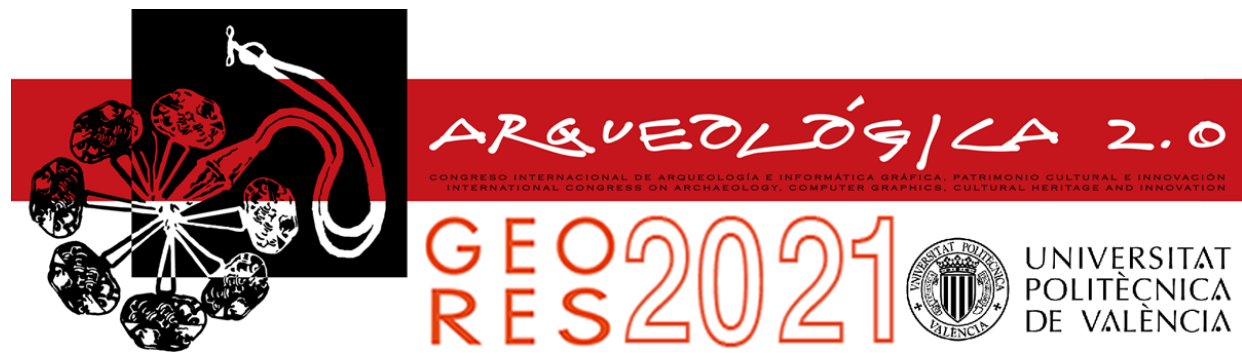

Proceedings of the joint international event $9^{\text {th }}$ ARQUEOLÓGICA

$2.0 \& 3^{\text {rd }}$ GEORES

Valencia (Spain).

26-28 April 2021

Received: 27/11/2020

Accepted: $17 / 03 / 2021$

DOI: https://doi.org/10.4995/Arqueologica9.2021.12133

\title{
THE LAST DECADE DEVELOPMENT OF PROJECT DATA MANAGEMENT THROUGH THE BIM
}

\author{
Francesco Di Stefano \\ Dipartimento di Ingegneria Civile, Edile e Architettura (DICEA), Università Politecnica delle Marche, Via Brecce Bianche 12, 60131 \\ Ancona, Italy. f.distefano@pm.univpm.it
}

\begin{abstract}
:
In the last decade, the management of data through information systems has become more widespread in the field of AEC. BIM represents a complete and integrated methodological approach based on a new process of modelling and interpreting reality into an information system. Starting from the data acquisition and collection, an initial geometric 3D model of the building can be designed. The lack of a direct connection between different information systems (e.g. BIM and GIS) for the same object forces to determine a common domain through the definition of an ontology. This semantic ontology will represent the basis of this knowledge model based on data coming from different information systems allowing data standardization and data enrichment operations. Thanks to the ontology, the threshold of interoperability between BIM and other information systems in a semantic web environment can be overcome. After a process of data conversion in exchange data format, a graph database can be built which collects all the heterogeneous data of the whole project. Ensuring access to anyone to the web graph database, it is possible to formulate a query for information recognition.
\end{abstract}

Keywords: geomatics, data management, BIM, ontology, interoperability, semantic web

\section{Introduction}

In the last decade, the management of data through information systems has become more widespread in the field of AEC (Architecture, Engineering and Construction) industry. Building Information Modelling (BIM) today represents a complete and integrated methodological approach based on a new process of modelling and interpreting reality into an information system. In short, BIM applications make it possible to: insert, in addition to 3D dimensional data, additional information useful for evaluating and analysing the project/model; manage and organise model objects parametrically; automatically update the relational database all the information about the building and the flow of data exchange between the actors involved in the construction process.

The advanced 3D model consists of individual building elements represented by 'intelligent' objects, i. e. objects that 'know' what they are and are associated with information describing their properties in relation to other objects. In several countries characterised by the progress of information technology, ad hoc regulations have been issued concerning and encouraging the use of such multi-data, multi-user and multi-purpose management platforms as BIM (Bradley, Li, Lark, \& Dunn, 2016).

While the use of parametric modelling for new buildings is becoming a stable reality, the paradigm of parametric modelling on historic buildings is slow to become established. There are several reasons for this, mainly related to the intrinsic characteristics of a historic building and the lack of a life-cycle concept.

HBIM (Murphy, McGovern \& Pavia, 2009) is a new way of modelling historic or existing building structures using a BIM process. HBIM is therefore a process that greatly increases the potential of the BIM method, because it also expands its use to the creation of models of existing buildings, and not only as their digital and geometric restitution in 3D, but as intelligent models rich in information, in which all the parts that compose them are objects with well-defined semantics. With HBIM, a kind of 'reverse engineering' is applied because, while modelling the building, the processes that led to the construction of that particular building organism are studied (López, Lerones, Llamas, Gómez-García-Bermejo \& Zalama, 2018).

Nowadays, there are several established geomatic techniques available, but for analysing historical assets, not all methodologies are always suitable. In particular, there are several factors to take into account, such as the accuracy and reliability of the survey, the adherence of the model to reality, the time and management of the historical data and so on.

Once initial parametric modelling of the components that make up the existing building been carried out, the challenge is to understand how to structure the data from a semantic point of view and to adopt a technique for standardization of the data. It is therefore necessary to define domains that help to determine a semantic hierarchy of the various entities and the relationships 
between them and the attributes associated with them. This introduces the concept of ontology, which is an innovative element that promotes data interoperability between a BIM platform and other information systems. For example, a building stands on terrain, so it has its own geolocation, but BIM software does not have the right tools to represent the terrain and define the buildingterrain relationship. The support of a geographic information system (GIS) becomes necessary in order to have a more complete representation of the context of this existing building. In this case, the ontology plays a decisive role in ensuring that there is a correspondence of the same data represented in different information systems that are characterised by a different semantic structure.

Interoperability between different information systems can be ensured with web-based tools. This will make it possible to produce information that can be made accessible by any user, thus defining so-called open data.

Concerning the interoperability between BIM and GIS, information systems are taken as an example for this research, it can be said that there are already software manufacturers such as ESRI and Autodesk that are implementing an integrated BIM-GIS platform (ESRI, 1969 ) but it is necessary to understand the dynamics that allow to operate in this extended platform and to improve this dual interoperability.

\section{Objectives of the research}

In this perspective, the objectives of the research project, on which it focuses most in order to highlight the innovative aspects linked to the development of the data management system using BIM, are as follows:

- Optimization of survey data through advanced geomatics techniques for 3D modelling;

- Semantic ontology as a common domain between BIM and other information management systems;

- Interoperability and data exchange between BIM and other information management systems.

\section{Critical literature review}

The integration of geomatics techniques is particularly relevant for accelerating spatial data collection from existing buildings (Gómez-García-Bermejo, Zalama \& Feliz, 2013). The accuracy can be ensured after a data optimization process (Akbarnezhad, Ong \& Chandra, 2014). Some research projects have been focused on optimizing modelling times (Biagini, Capone, Donato \& Facchini, 2016), combining data from the geomatic survey into BIM by the creation of parametric objects directly from the 3D point cloud, defining the most suitable levels of accuracy of the parametric model and characterizing it with data related to materials and construction techniques (Garagnani \& Manferdini, 2013; Spallone, Piano, A. \& Piano, S., 2016).

BIM software describes 3D geometry and identifies semantic relationships between building components basing on the hierarchical structure given by the software used. Some limitations of BIM software on data management, in comparison to other information systems, are linked to a lack of a defined LOD (Level of
Detail) structure. It needs to provide first an external wellstructured database, but it is often not easy to import it into BIM, especially for existing buildings. An example of scheme characterized by a semantic structure based on LOD can be offered by GIS (or better CityGML) (OGC, 1994). GIS is another information system that is well suited for cataloguing building data, but not only that, it also allows the building to be related to the terrain on which it stands. This is a further advantage over BIM.

Having said that, there are some dissimilarities between BIM and GIS, such as spatial scale, level of representation of geometric models and structure of database. In computer science, ontologies are adopted as domains providing a formal structure for sharing and managing data defining objects (taxonomies) and their relationships (El-Diraby, Lima \& Feis, 2005).

Ontologies are used to overcome the barriers to heterogeneous semantic data sharing (Hor, 2015). The definition of a semantic ontology as a common domain, for sharing and managing data coming from different information system, represents a valuable solution promoting a multidirectional approach between BIM and other information systems, such as GIS (Deng, Cheng \& Anumba, 2016).

The integration of data coming from different information modelling environment represents an efficient way for defining a smarter, more sustainable and resilient project. It gives the possibilities to combine heterogeneous data: geometric shapes, quantitative analysis, enrichment of semantic knowledge, application of different technologies and multi-scale management (Ma \& Ren 2017; Fosu, Suprabhas, Rathore \& Cory, 2015).

Data exchange between BIM and other information systems is ensured through interoperability, which is only possible through the web environment. While, syntactic interoperability refers to the use common data format to exchange information between BIM and GIS systems, using a domain of one of the information systems, a bidirectional approach is ensured (Deng, Cheng \& Anumba, 2016) through dual semantic interoperability between information systems. Examples of syntactic interoperability are systems that combine building data with landscape maps, data formats of BIM object on GIS environment, and solutions to convert BIM data from IFC (Industry Foundation Class) to CityGML (Kolbe, Gröger \& Plümer, 2005; Karimi \& Akinci, 2009; De Laat \& Van Berlo, 2011). The highest level of interoperability is guaranteed by the semantic aspect of data integration. The key point of semantic interoperability is to make sure that the features and relations between information management systems are maintained during data conversion (Peachavanish, Karimi, Akinci, \& Boukamp, 2006).

The semantic web technology represents new efficient online platform to make possible this kind of interoperability. The web ontology language (OWL) expresses the data in terms of classes. A collection of these classes, their attributes and relations can be stored as RDF triples describing each individual object, its properties and features, which can be understood as a graph-based on nodes (entities) and vertices (relationships) (Karan \& Irizarry, 2015; Hor, Jadidi \& Sohn, 2016). Taking this approach into account, it becomes possible to move forward a graph database 
direction, useful also for a BIM data interaction providing so a complete interoperability in the web environment and so allowing an eventually bidirectional way of data transfer between information systems.

\section{Materials and methods}

Starting from the data acquisition and collection, an initial geometric 3D model of the building can be designed, but first, some considerations must be taken into account. It needs to understand on which scale of detail to represent the various elements that make up the building structure according to the BIM scheme, how non-geometrical attributes can be linked to the 3D model and then how the building can be connected to the terrain where it stands.

First, a conceptual model is developed, which is then converted into a relational database to represent the basis of the semantic structure adopted for the BIM project. On the other hand, with the elaboration of a 3D model of the terrain in GIS environment, one tries to establish which LOD to operate at the building scale in order to find elements in common with those modelled in BIM.

The lack of a direct connection between these two information systems forces us to determine a common domain through the definition of an ontology.

This semantic ontology will represent the basis of this knowledge model based on data coming from different information systems allowing data standardization and data enrichment operations.

Thanks to the ontology, the threshold of interoperability between BIM and other information system in a semantic web environment can be overcome.

After a process of data conversion in exchange data format, a graph database can be built which collects all the heterogeneous data of the whole project. Ensuring access to anyone to the web graph database, also nonexperts, it is possible to formulate a query for the information recognition. A further step could be the link to a digital twin model.

The following flowchart summarizes the methodology of the research project (Fig. 1).

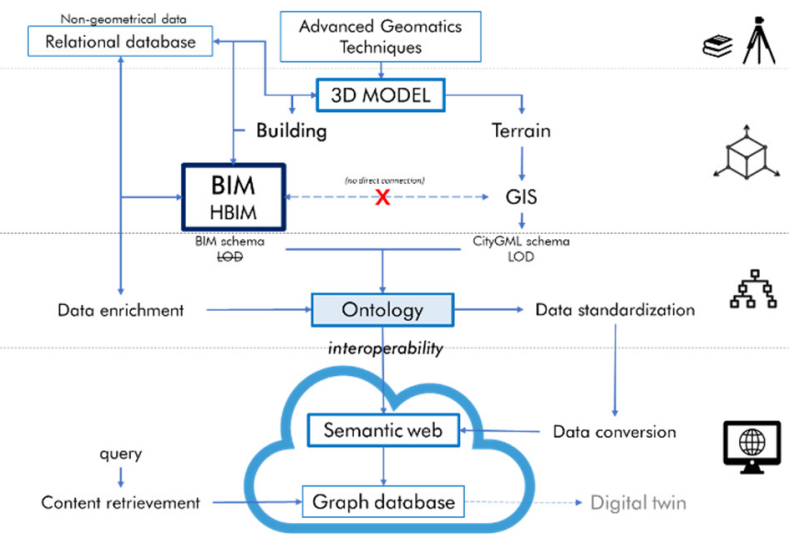

Figure 1: Workflow of the research project.

For the purpose of this research, existing, historical buildings or newly built artefacts, building complexes and small villages were taken as case studies. Not only the buildings are considered, but also their location, such as the ground on which they stand, in order to define relationships not only between elements of the same building but also with other buildings and the environment in which they are located.

\section{Partial results}

\subsection{Advanced geomatics techniques}

The improvement in technical skills with geomatics instruments by the PhD Student, led him to assess the data acquisition in different type of built environments. In particular, Mobile Laser Scanning (MLS), Mobile Mapping System (MMS) and devices with SLAM technology integrated to other geomatics techniques, when they occurred, has been carried out in different environments: structured outdoor environment (Di Stefano, Chiappini, Piccinini, \& Pierdicca, 2019), indoor cluttered environment (Paolanti et al., 2019) and tight underground environment. During the data processing phase, when the data obtained from different acquisition techniques are combined, the assessment of the final resolution is evaluated, just to ensure the higher accuracy of the final point cloud (Fig. 2) useful for the following 3D model design.

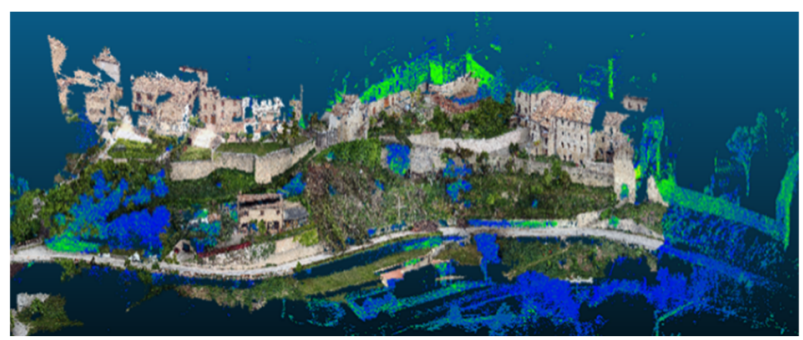

Figure 2: Example of integration and assessment of advanced geomatics technologies (city wall of San Ginesio, Italy).

The objective of this first part of the research activity is not to create a detailed 3D geometrical model, for example for the definition of parametric surfaces, but only to represents the volume of $3 \mathrm{D}$ objects for the management of information linked to them. In addition to modelling the buildings, the terrain is also modelled in GIS by obtaining DTM (or DSM) from the survey data (Fig. 3).

\subsection{Semantic ontology}

For each case study analysed, a conceptual model was drafted to identify the objects that would be created in the BIM modelling phase and to define the initial relationships between the objects and their components. Some BIM software does not allow each object to be named, so an identification system (ID-code) is created externally to classify it in the database within the software and make it easy to recognise (Di Stefano, Malinverni, Pierdicca, Fangi \& Ejupi, 2019).

In order to develop a strategy for building knowledge modelling to actively support the project process, it proposes a dual approach: one ontology-based to represent the semantic contents, especially nongeometrical information, one in BIM environment to represent the logic of the construction, above all geometrical-constructive aspects (Di Stefano, Gorreja, Malinverni \& Mariotti, 2020).

Some limitations of BIM, as parametric software, don't allow a direct importing of semantic ontology scheme, 
from an external tool. The structure of BIM software not based on a well-defined LOD (e.g. family-type-instance of Autodesk Revit) make it hard to find a correspondence with the ontological scheme. Before re-define this tripartite ontological structure in BIM software, it occurs to identify the domains that compose it. Taking as an example the case study of a historic building the ontological scheme, useful for a preservation and restoration management project, is characterized by four main domains such as artefact, investigation process, actors and lifecycle (Fig. 3a). This empirical representation (ontology) combined with the visual and descriptive 3D model provides a knowledge modelling of the historical artefact (Fiorani \& Acierno, 2017). The BIM model can be also enriched with thematic mapping that represents an adding information to the surfaces of the 3D object itself (e.g. decay mapping) (Malinverni, Mariano, Di Stefano, Petetta, \& Onori, 2019) (Fig. CityGML 3b). Then, through specific plug-in tools of BIM software, non-geometrical information, such as the glossaries of building regulations (e.g. decay glossaries), can be imported (Di Stefano, Gorreja, Malinverni \& Mariotti, 2020) (Fig. 3c).

The loading of digital libraries into BIM software allows rapid conversion of data into an interchange format. IFC (BuildingSMART, 2005) is the basic component of openBIM, the online BIM data management platform.

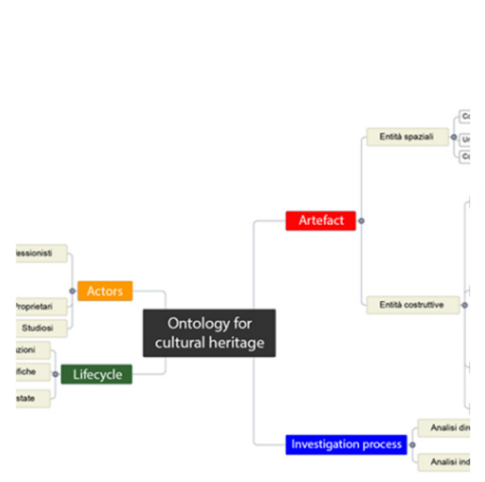

(a)

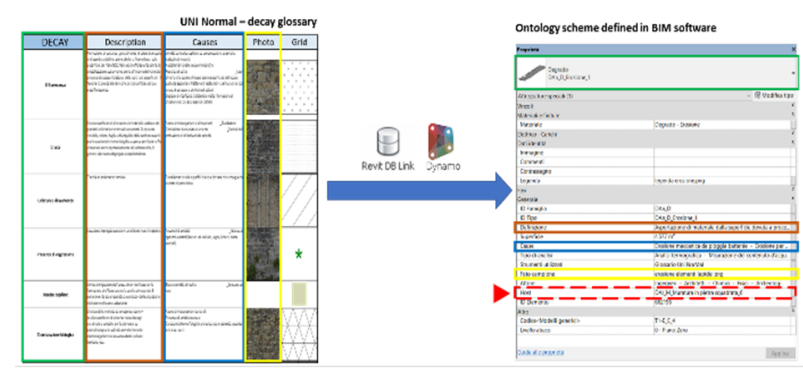

(c)

Figure 3: Management of information linked to 3D objects: a) A semantic ontology for a historical building; b) Thematic mapping on a BIM model (e.g. decay mapping); c) Conversion of a glossary into ontological scheme in BIM software.

\subsection{Interoperability and data exchange}

Up to now, it has been talking about BIM dealing with the management of data about a building, now the aim is to relate the building itself to the terrain on which it stands. GIS is the information system that best processes spatial data, allowing 3D models to be created at different scales of detail. The fictitious volume of the same building analysed in BIM is also created on the DTM (Fig.4), so as to have a common element that can link information from different information systems.

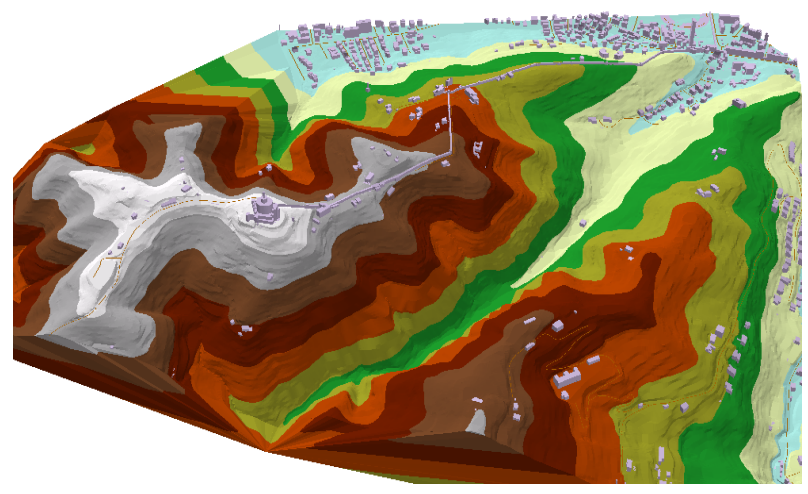

Figure 4: DTM with buildings (LOD 1).

But the lack of a direct connection between information systems like BIM and GIS does not allow to ensure the right information exchange. The solution is to adapt the ontology also for GIS data, enriching it, finding correspondences and creating relationships with BIM elements. The GIS ontology can be developed basing on CityGML scheme (OGC, 1994).

The creation of 3D GIS model of the same object analysed on BIM environment and its transformation in online format, CityGML, has been executed (Fig. 4). This process allowed to make a comparison between the CityGML scheme based on LOD and the BIM one based only on the tripartition structure, as previously mentioned. A process of data standardisation is necessary in order to identify common elements and determine correspondences. This leads to the creation of an integrated BIM-GIS ontology scheme (Fig. 5).

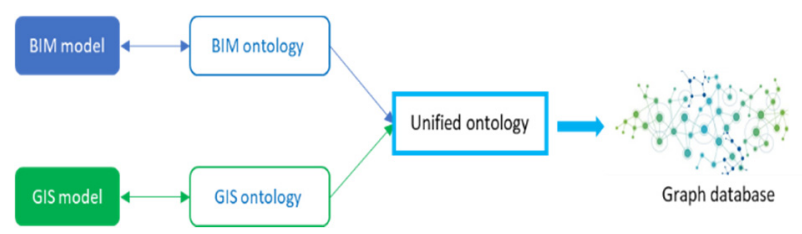

Figure 5: BIM-GIS ontology integration.

This unified ontological schema overcomes the threshold of interoperability between BIM and GIS through the definition of nodes that form the basis of the graph database created in the semantic web environment. This requires the conversion of BIM and GIS data into RDF triples. While BIM is already provided with digital libraries where IFC data are easily to convert to RDF format, transforming CityGML data in the same format required a longer process. According to the file formats supported by some graph databases, CityGML data has been converted into GeoJSON (OGC, 1994; Malinverni \& al., 2020).

Finally, obtained all data into lightweight interchange format, the ontological scheme can be directly transformed into a graph database. Graph databases belong to NoSQL databases, useful to store unstructured information. After comparing several graph database 
examples, ArangoDB was chosen for this research project (Fernandes \& Bernardino, 2018).

ArangoDB (ArangoDB, 2011) is a graph database based on documents and edges. The former store information, whereas the latter define relationships between data (Fig. $6)$.

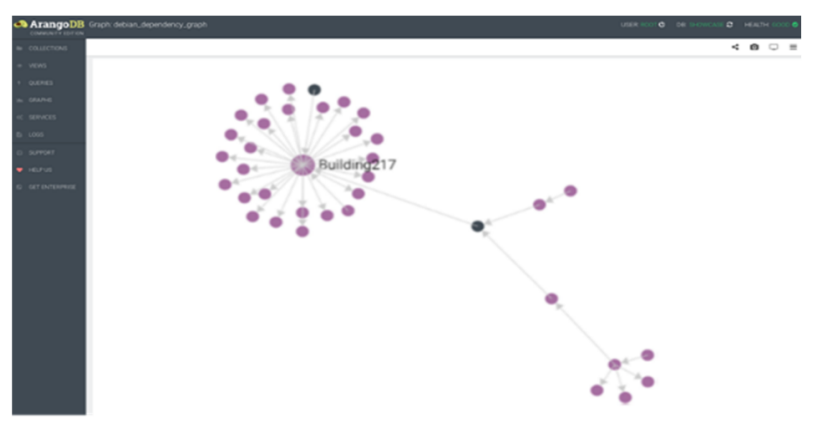

Figure 6: Example of a graph database created in the semantic web environment (Arango DB).

\section{Conclusions and future works}

Information management systems, related to building and urban planning, represent today a fundamental tool in both working and research fields. However, the exchange of data between information systems is still a huge challenge, while, if possible, it could bring enormous benefits. To guarantee the interoperability of data between BIM and other information systems (such as
GIS) it needs to use tools already standardized and accessible to everyone, which means basing on defined and known schemes and ontologies. Semantic web technology is used to get interpretable transferred data by both BIM and GIS environment.

The current main achievements of this research project are listed as follow:

- Assessment of the accuracy of acquired data after integration of different advanced geomatics technologies;

- Definition of semantic ontology for a building project;

- Overcoming of the limitations of BIM software to import an ontological scheme;

- 3D modelling in GIS environment defining the connection building-terrain;

- Definition of an integrated BIM-GIS ontology;

- GIS data conversion in RDF format through FME;

- A first attempt at graph database creation based on RDF triples obtained from GIS data.

In order to complete the PhD research, the planned activities are: check of the dual interoperability BIM-GIS through the graph database and testing the query formulation for content retrievement. Finally, a tentative approach to the digital twin model will be made.

\section{References}

Akbarnezhad, A., Ong, K. C. G., \& Chandra, L. R. (2014). Economic and environmental assessment of deconstruction strategies using building information modeling. Automation in Construction, 37, $131-144$. https://doi.org/10.1016/j.autcon.2013.10.017

ArangoDB. (2011). Arango DB Oasis. Retrieved July 15, 2019, from https://www.arangodb.com/

Biagini, C., Capone, P., Donato, V., \& Facchini, N. (2016). Towards the BIM implementation for historical building restoration sites. Automation in Construction, 71, 74-86. https://doi.org/10.1016/j.autcon.2016.03.003

Bradley, A., Li, H., Lark, R., \& Dunn, S. (2016). BIM for infrastructure: An overall review and constructor perspective. Automation in Construction, 71, 139-152. https://doi.org/10.1016/j.autcon.2016.08.019

BuildingSMART. (2005). Industry Foundation Classes (IFC). Retrieved July 15, 2019, from https://www.buildingsmartitalia.org/standard/standard-bs/industry-foundation-classes-ifc/

Deng, Y., Cheng, J.C. \& Anumba, C. (2016). Mapping between BIM and 3D GIS in different levels of detail using schema mediation and instance comparison. Automation in Construction, 67, 1-21 https://doi.org/10.1016/j.autcon.2016.03.006

De Laat, R., \& Van Berlo, L. (2011). Integration of BIM and GIS: The development of the CityGML GeoBIM extension. In Advances in 3D geo-information sciences (pp. 211-225). Springer, Berlin, Heidelberg. https://doi.org/10.1007/9783-642-12670-3_13

Di Stefano, F., Chiappini, S., Piccinini, F., \& Pierdicca, R. (2019). Integration and Assessment Between 3D Data from Different Geomatics Techniques. Case Study: The Ancient City Walls of San Ginesio (Italy). In International Workshop on R3 in Geomatics: Research, Results and Review (pp. 186-197). Springer, Cham. https://doi.org/10.1007/978-3-030-62800-0_15

Di Stefano, F., Malinverni, E. S., Pierdicca, R., Fangi, G., \& Ejupi, S. (2019). HBIM implementation for an ottoman mosque. Case of study: Sultan Mehmet Fatih ii mosque in Kosovo. International Archives of the Photogrammetry, Remote Sensing \& Spatial Information Sciences, 42, 429-436. https://doi.org/10.5194/isprs-archives-XLII-2-W15-429-2019

Di Stefano, F., Gorreja, A., Malinverni, E. S., \& Mariotti, C. (2020). Knowledge modeling for heritage conservation process: from survey to HBIM implementation. The International Archives of Photogrammetry, Remote Sensing and Spatial Information Sciences, 44, 19-26. https://doi.org/10.5194/isprs-archives-XLIV-4-W1-2020-19-2020

El-Diraby, T. A., Lima, C., \& Feis, B. (2005). Domain taxonomy for construction concepts: toward a formal ontology for construction knowledge. Journal of computing in civil engineering, 19(4), $394-406$. 
https://doi.org/10.1061/(ASCE)CO.1943-7862.0000646

ESRI (1969). Autodesk \& Esri - Collaboration Connects GIS and BIM. Retrieved March 11, 2019, from https://www.esri.com/en-us/about/esri-partner-network/our-partners/global-alliances/autodesk-esri

Fiorani, D., \& Acierno, M. (2017). Conservation process model (CPM): a twofold scientific research scope in the information modelling for cultural heritage. International Archives of the Photogrammetry, Remote Sensing \& Spatial Information Sciences, 42, 283-290. https://doi.org/10.5194/isprs-archives-XLII-5-W1-283-2017.

Fernandes, D., \& Bernardino, J. (2018). Graph Databases Comparison: AllegroGraph, ArangoDB, InfiniteGraph, Neo4J, and OrientDB. In DATA (pp. 373-380). https://doi.org/10.5220/0006910203730380

Fosu, R., Suprabhas, K., Rathore, Z., \& Cory, C. (2015). Integration of Building Information Modeling (BIM) and Geographic Information Systems (GIS)-a literature review and future needs. In Proceedings of the 32nd CIB W78 Conference, Eindhoven, The Netherlands (pp. 27-29).

Garagnani, S., \& Manferdini, A. M. (2013). Parametric accuracy: building information modeling process applied to the cultural heritage preservation. International Archives of the Photogrammetry, Remote Sensing and Spatial Information Sciences, 5(1), 87-92. https://doi.org/10.5194/isprsarchives-XL-5-W1-87-2013

Gómez-García-Bermejo, J., Zalama, E., \& Feliz, R. (2013). Automated registration of 3D scans using geometric features and normalized color data. Computer-Aided Civil and Infrastructure Engineering, 28(2), 98-111. https://doi.org/10.1111/j.1467-8667.2012.00785.x

Hor, A. H. (2015). A semantic Web platform for BIM-GIS integration. https://doi.org/10.13140/RG.2.1.4176.6643

Hor, A. H., Jadidi, A., \& Sohn, G. (2016). BIM-GIS integrated geospatial information model using semantic web and RDF graphs. ISPRS Ann. Photogramm. Remote Sens. Spat. Inf. Sci, 3(4), 73-79. https://doi.org/10.5194/isprs-annals-III4-73-2016

Karan, E. P., \& Irizarry, J. (2015). Extending BIM interoperability to preconstruction operations using geospatial analyses and semantic web services. Automation in Construction, 53, 1-12. https://doi.org/10.1016/j.autcon.2015.02.012

Karimi, H. A., \& Akinci, B. (2009). CAD and GIS integration. CRC Press.

Kolbe, T. H., Gröger, G., \& Plümer, L. (2005). CityGML: Interoperable access to 3D city models. In Geo-information for disaster management (pp. 883-899). Springer, Berlin, Heidelberg. https://doi.org/10.1007/3-540-27468-5_63

López, F. J., Lerones, P. M., Llamas, J., Gómez-García-Bermejo, J., \& Zalama, E. (2018). A review of heritage building information modeling (H-BIM). Multimodal Technologies and Interaction, 2(2), 21. https://doi.org/10.3390/mti2020021

Ma, Z., \& Ren, Y. (2017). Integrated application of BIM and GIS: an overview. Procedia Engineering, 196, $1072-1079$. https://doi.org/10.1016/j.proeng.2017.08.064

Malinverni, E. S., Mariano, F., Di Stefano, F., Petetta, L., \& Onori, F. (2019). Modelling in HBIM to document materials decay by a thematic mapping to manage the cultural heritage: the case of "Chiesa della Pietà" in Fermo. International Archives of the Photogrammetry, Remote Sensing \& Spatial Information Sciences. https://doi.org/10.5194/isprsarchives-XLII-2-W11-777-2019

Malinverni, E. S., Naticchia, B., Garcia, J. L. L., Gorreja, A., Uriarte, J. L., \& Di Stefano, F. (2020). A semantic graph database for the interoperability of 3D GIS data. Applied Geomatics, 1-14. https://doi.org/10.1007/s12518-02000334-37

Murphy, M., McGovern, E., \& Pavia, S. (2009). Historic building information modelling (HBIM). Structural Survey, $311-327$. https://doi.org/10.1108/02630800910985108

OGC. (1994). Open Geospatial Consortium. Retrieved July 15, 2019, from https://www.opengeospatial.org/standards/citygml

Paolanti, M., Pierdicca, R., Martini, M., Di Stefano, F., Morbidoni, C., Mancini, A., Christian Morbidoni, C., Malinverni, E.S., Frontoni, E., \& Zingaretti, P. (2019). Semantic 3D object maps for everyday robotic retail inspection. In International Conference on Image Analysis and Processing (pp. 263-274). Springer, Cham. https://doi.org/10.1007/978-3-03030754-7_27

Peachavanish, R., Karimi, H. A., Akinci, B., \& Boukamp, F. (2006). An ontological engineering approach for integrating $\mathrm{CAD}$ and GIS in support of infrastructure management. Advanced Engineering Informatics, 20(1), 71-88. https://doi.org/10.1016/j.aei.2005.06.001

Spallone, R., Piano, A., \& Piano, S. (2016). BIM and cultural heritage: multi-scalar and multi-dimensional analysis and representation of an historical settlement. DisegnareCON, 9(16), 13-1. 